\title{
纳米晶体纤维素增强磷酸钙骨水泥的研究
}

\author{
赵军胜 ${ }^{1}$, 屈树新 ${ }^{1}$, 黄 萍 ${ }^{1}$, 刘宗光 ${ }^{1}$, 王铈汶 ${ }^{1,2}$, 翁 杰 $^{1}$
}

(1. 西南交通大学 材料科学与工程学院, 材料先进技术教育部重点实验室, 成都 610031 ; 2. 国家纳米科学中心, 中国科学院纳米生物安全性与生物效应重点实验室，北京 100190)

摘 要: 本研究探索具有良好力学性能的纳米晶体纤维素( NCC)对磷酸钻骨水泥(CPC)抗压强度的影响。采用万能力 学试验机、Gilmore 双针、X 射线衍射仪(XRD)和 X 射线光电子能谱仪(XPS)表征含不同 NCC 的 CPC 理化性能; 利 用扫描电子显微镜(SEM)和荧光显微镜观察 CPC 断面形貌和荧光标记的 NCC 在 CPC 中的分散。抗压强度结果表 明: NCC 能显著提高 CPC 的抗压强度, 且 $2 \% \mathrm{NCC}-\mathrm{CPC}$ 的抗压强度最高, 约为 $27 \mathrm{MPa}$; CPC 的凝固时间随 NCC 含 量的增加而延长, 含量为 $2 \%$ 时基本符合临床要求; XRD 和 XPS 结果显示 NCC 与 $\mathrm{Ca}^{2+}$ 形成不稳定的配合物, 促进 了 $\mathrm{CPC}$ 中二水磷酸氢钲(DCPD)和 $\mathrm{CaCO}_{3}$ 的溶解和转化; SEM 观察结果显示加入 $\mathrm{NCC}$ 使 $\mathrm{CPC}$ 内部结构更致密, 孔 隙和裂纹减少; 荧光显微观察结果表明 NCC 在 CPC 中均匀分散。

关 键 词: 磷酸钻骨水泥; 纳米晶体纤维素; 抗压强度; 分散性

中图分类号: TB321; O611; Q539 文献标识码: A

\section{Calcium Phosphate Cement Reinforced by Nanocrystalline Cellulose}

\author{
ZHAO Jun-sheng ${ }^{1}$, QU Shu-Xin ${ }^{1}$, HUANG Ping ${ }^{1}$, LIU Zong-Guang ${ }^{1}$, WANG Shi-Wen ${ }^{1,2}$, WENG Jie ${ }^{1}$ \\ (1. Key Lab of Advanced Technologies of Materials, Ministry of Education, School of Material Science and Engineering, \\ Southwest Jiaotong University, Chengdu 610031, China; 2. Key Laboratory for Biomedical Effects of Nanomaterials and \\ Nanosafety, Chinese Academy of Sciences, National Center for Nanoscience and Technology, Beijing 100190, China)
}

\begin{abstract}
The aim of this study is to investigate the effect of nanocrystalline cellulose (NCC) with favorable mechanical properties on the compressive strength of calcium phosphate cement (CPC). Compressive test, Gilmore needle test, X-ray diffraction (XRD) and X-ray photoelectron spectroscopy (XPS) were used to analyze the physicochemical properties of CPC influenced by different contents of NCC. Scanning electron microscope (SEM) and fluorescence microscope were used to observe the morphologies of CPC and the dispersity of labeled-NCC in CPC, respectively. NCC significantly increased the compressive strength of CPC up to about $27 \mathrm{MPa}$ when the content of NCC was $2 \%$. Setting times of CPC were prolonged with increased NCC, but still met the clinical requirements when the content of NCC was not more than $2 \%$. XRD and XPS indicated that the combination of NCC with $\mathrm{Ca}^{2+}$ could form unstable coordination compound and NCC promoted the dissolution and conversion of dicalcium phosphate dehydrate (DCPD) and $\mathrm{CaCO}_{3}$. SEM showed that $\mathrm{CPC}$ became denser with fewer pores and cracks by addition of NCC. Fluorescence microscope demonstrated the homogeneous dispersion of labeled-NCC in CPC.
\end{abstract}

收稿日期：2014-07-25; 收到修改稿日期：2014-09-17

基金项目：973 项目(2012CB933602); 国家自然科学基金(51372210); 教育部博士点基金(20130184110023); 四川省高校科 研创新团队建设计划项目(14TD0050)

National Basic Research Program of China (973 Program, 2012CB933602); National Natural Science Foundation of China (51372210); Research Fund for the Doctoral Program of Higher Education of China (20130184110023); Construction Program for Innovative Research Team of University in Sichuan Province (14TD0050)

作者简介: 赵军胜(1987-), 男, 硕士研究生. E-mail: junshengz0901@163.com

通讯作者：屈树新，教授. E-mail: qushuxin@swjtu.edu.cn 
Key words: calcium phosphate cement; nanocrystalline cellulose; compressive strength; dispersity

磷酸钙骨水泥(Calcium phosphate cement, CPC) 因具有良好的生物相容性、可生物降解性和可任意 塑形等优点, 已被用于临床骨替换材料 ${ }^{[1-3]}$ 。但 CPC 力学强度较低, 仅能用于非负重部位 ${ }^{[4-5]}$ 。因此, 提 高 $\mathrm{CPC}$ 的力学强度具有重要的意义。

天然纤维素(cellulose)来源于自然界, 具有可再 生、可生物降解、无毒等特点。天然纤维素由排列 紧密、性能稳定的结晶区和结构疏松、易降解的无 定形区随机分布构成。在酸或酶解下，去除天然纤维 素中的无定型区而保留结晶区, 可得到具有高强度 (抗张强度 $7500 \mathrm{MPa}$ )、高杨氏模量(100 140 GPa)、 高比表面积 $\left(150 \sim 250 \mathrm{~m}^{2} / \mathrm{g}\right)$ 的纳米晶体纤维素 (Nanocrystalline cellulose, NCC) $)^{[6-9]}$ 。 NCC 还具有高 亲水性、良好的生物相容性和生物可降解性等优点, 因此常作增强剂应用于复合材料的研究 ${ }^{[7,10-11]}$ 。但 $\mathrm{NCC}$ 在 CPC 中的应用尚未见报道。因此, 本研究利 用 NCC 优良的力学性能, 研究 NCC 对 CPC 抗压强 度的影响, 并探索其影响机理。

\section{1 实验方法}

\section{$1.1 \mathrm{NCC} / \mathrm{CPC}$ 的制备}

羟基磷灰石 $\left(\mathrm{Ca}_{10}\left(\mathrm{PO}_{4}\right)_{6}(\mathrm{OH})_{2}, \mathrm{HA}\right)$ 和 $\alpha$-磷酸三 钻 $\left(\alpha-\mathrm{Ca}_{3}\left(\mathrm{PO}_{4}\right)_{2}, \alpha-\mathrm{TCP}\right)$ 购自四川大学生物材料工程 研究中心; NCC 由国家纳米科学中心蒋兴宇研究员 课题组惠赠, 二水磷酸氢钙 $\left(\mathrm{CaHPO}_{4} \cdot 2 \mathrm{H}_{2} \mathrm{O}, \mathrm{DCPD}\right)$ 由本实验室合成 ${ }^{[12]}$; 其余试剂均为分析纯, 购自成 都科龙化工试剂厂。

选用 Biocement D 配方 ${ }^{[13]}$, 固相包括 $\alpha$-TCP $(58 \mathrm{wt} \%) 、 \mathrm{DCPD}(25 \mathrm{wt} \%) 、 \mathrm{HA}(8.5 \mathrm{wt} \%)$ 和 $\mathrm{CaCO}_{3}$ $(8.5 \mathrm{wt} \%)$ 。液相为磷酸盐缓冲液 $(\mathrm{PBS}), \mathrm{pH}$ 为 7.4 。 液固比为 $0.3 \mathrm{~mL} / \mathrm{g}$ 。在液相中加入分别占 $\mathrm{CPC}$ 粉末 质量 $2 \%$ 和 $4 \%$ 的 NCC, 超声分散。将 CPC 粉末与 液相调和后用不锈钢模具成型, 置于 $37{ }^{\circ} \mathrm{C}$ 、相对 $100 \%$ 湿度的环境中水化 $24 \mathrm{~h}$, 制备含 NCC 的 CPC, 不含 $\mathrm{NCC}$ 的 $\mathrm{CPC}$ 为空白对照, 分别标记为 $2 \%$ NCC-CPC、 $4 \%$ NCC-CPC 和 CPC。

\subsection{NCC 的荧光标记}

根据文献[14], 在 $1 \mathrm{wt} \%$ 的 NCC 悬浮液中加入 $0.1 \mathrm{mg} / \mathrm{mL}$ 的 7-氨基-4-甲基香豆素(AMC, Ruibio, Germany)溶液, 于黑暗、 $37^{\circ} \mathrm{C}$ 和 $\mathrm{pH}=8.0$ 条件下反应 $24 \mathrm{~h}$, 纯化干燥得到 $\mathrm{AMC}$ 标记的 NCC。

\section{3 性能表征}

\subsubsection{CPC 抗压强度测试}

采用万能力学试验机(5567, Instron, USA)测定 各试样的抗压强度, 样品尺寸为 $\phi 6 \mathrm{~mm} \times 12 \mathrm{~mm}$, 加 载速度为 $0.5 \mathrm{~mm} / \mathrm{min}$, 每组测试 6 个样品。

\subsubsection{CPC 凝固时间测试}

采用 Gilmore 双针法测量各组 CPC 的初凝时间 (Initial time, IT) 和终凝时间(Final time, FT) ${ }^{[15]}$, 每组 测试 3 个样品。

\subsubsection{CPC 物相分析}

将水化 $24 \mathrm{~h}$ 的各组 CPC 试样干燥, 研磨, 进行 $X$ 射线衍射(XRD, $\chi$ 'Pert Pro, Philips, Netherlands)测 定。铜靶, 电压 $40 \mathrm{kV}$, 电流 $40 \mathrm{~mA}$, 扫描范围: $2 \theta=4^{\circ} \sim 60^{\circ}$ 。

\subsubsection{CPC 表面元素分析}

采用 $\mathrm{X}$ 射线光电子能谱仪(XPS, XSAM800, USA)分析 CPC、 $2 \%$ NCC-CPC 和 4\% NCC-CPC 试样 表面元素的组成及化学态。 $\mathrm{Al}$ 靶, 测试电压: $12 \mathrm{kV}$, 测试电流: $11 \mathrm{~mA}$ 。

\subsection{5 微观形貌表征}

取 CPC、 $2 \%$ NCC-CPC 和 4\% NCC-CPC 试样的 自然断面，喷金后进行扫描电子显微镜 (SEM, FEI-Quanta200, Philips, Netherlands)观察。

取数滴 $0.01 \mathrm{wt} \%$ 的 $\mathrm{NCC}$ 分散液滴于载有碳膜的 铜网上, 再滴加数滴 $1 \mathrm{wt} \%$ 的磷铇酸溶液对 $\mathrm{NCC}$ 进 行负染, 晾干后, 使用场发射透射电子显微镜(TEM, JEM-2100F, Japan)观察其形貌。

\subsubsection{NCC 在 CPC 中分散性的研究}

制备含 7-氨基-4 甲基香豆素(AMC)荧光物标记 的 $\mathrm{NCC} / \mathrm{CPC}$ 试样 $(\phi 10 \mathrm{~mm} \times 5 \mathrm{~mm})$, 使用苂光显微 镜(BX63, Olympus, Japan)观察表征 NCC 在 CPC 中 的分布。

\subsection{7 统计学分析}

采用 Student's T test 对各组 CPC 试样的抗压 强度、凝固时间进行显著性差异分析。 $p<0.05$ 为显 著性差异; $p<0.01$ 为极显著性差异。

\section{2 结果与讨论}

\subsection{CPC 抗压强度测试}

图 1(a)为各组 CPC 样品水化 $24 \mathrm{~h}$ 的抗压强度。 $2 \%$ NCC-CPC 和 $4 \%$ NCC-CPC 的抗压强度分别为 

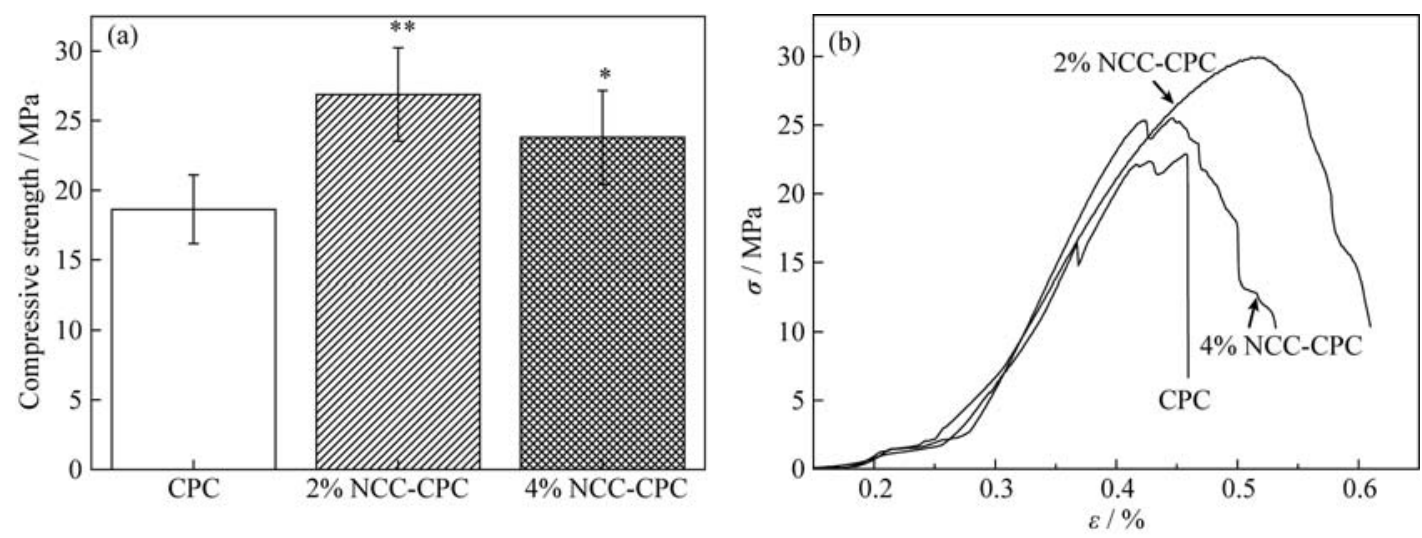

图 $1 \mathrm{CPC} 、 2 \% \mathrm{NCC}-\mathrm{CPC}$ 和 $4 \% \mathrm{NCC}-\mathrm{CPC}$ 水化 $24 \mathrm{~h}$ 后的抗压强度(a)和典型的应力一应变曲线(b)

Fig. 1 Compressive strength of CPC, $2 \%$ NCC-CPC and $4 \%$ NCC-CPC after setting for $24 \mathrm{~h}\left(n=6,{ }^{*} p<0.05\right.$, ** $\left.p<0.01\right)($ a) and the typical stress-strain curves (b)

(26.9 \pm 3.4$) \mathrm{MPa}$ 和 $(23.8 \pm 3.4) \mathrm{MPa}$ ，与 $\mathrm{CPC}$ 的相比分 别具有极显著性差异 $(p<0.01)$ 和显著性差异 $(p<0.05)$ 。图 1(b) 为各组 CPC 样品典型的应力-应变 曲线。CPC 呈现脆性断裂，而 $2 \%$ NCC-CPC 和 $4 \%$ NCC-CPC 呈现㓞性断裂, 同时断裂强度提高, 即加 入 $\mathrm{NCC}$ 提高了 $\mathrm{CPC}$ 的韧性和抗压强度。 $4 \%$ NCC-CPC 的抗压强度较 2\% NCC-CPC 的降低, 可 能是由于 $\mathrm{NCC}$ 的团聚作用导致应力集中 ${ }^{[16]}$; 此外, 因 NCC 具有良好的吸水溶胀性 ${ }^{[17]}$, 过多的 NCC 降 低了 $\mathrm{CPC}$ 的液固比, 使 CPC 在水化时部分颗粒无法 与水分充分接触, 水化反应不完全。

\section{$2.2 \mathrm{CPC}$ 的凝固时间}

图 2 为各组 $\mathrm{CPC}$ 的初凝和终凝时间。随 NCC 含 量的增加, CPC 的初凝和终凝时间均延长, 其中 $2 \%$ NCC-CPC 的初凝 (IT) 和终凝 (FT) 时间分别为 (9.0 \pm 0.4$) \mathrm{min}$ 和(14.5 \pm 1.4 )min, 基本满足临床上的需求, 即 $4 \min \leqslant \mathrm{IT} \leqslant 8 \min , 10 \mathrm{~min} \leqslant \mathrm{FT} \leqslant 15 \min ^{[18]} \mathrm{CPC}$ 凝 结过程的物理本质在于水化产物体积的增大导致

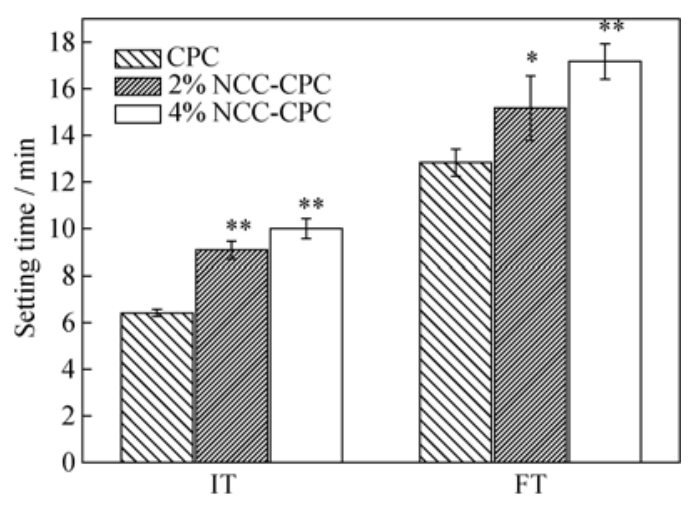

图 2 CPC、 $2 \%$ NCC-CPC 和 4\% NCC-CPC 的初凝和终凝时间 Fig. 2 Initial and final setting times of CPC, 2\% NCC-CPC and $4 \%$ NCC-CPC $(n=3, * p<0.05, * * p<0.01)$
CPC 颗粒间距离缩短, 直到颗粒间通过产物搭接。产 生结晶并接，使 $\mathrm{CPC}$ 浆体失去流动性而凝结 ${ }^{[19]}$ 。 $\mathrm{NCC}$ 可能会吸附在 CPC 颗粒表面, 掩盖 CPC 颗粒水化反 应的部分活性位点和增大 CPC 颗粒间的距离; 此外, NCC 的加入降低了液固比, 减慢 CPC 的水化速率, 以上两者均可延长 $\mathrm{CPC}$ 的凝固时间。

\section{$2.3 \mathrm{CPC}$ 物相分析}

图 3(a)为各组 CPC 水化 $24 \mathrm{~h}$ 的 XRD 图谱。各 组 CPC 试样的衍射峰主要为固相组成中的 $\alpha-\mathrm{TCP}$ 和 DCPD 以及少量的 $\mathrm{CaCO}_{3}$ 和 $\mathrm{HA}$, 且 $\mathrm{CPC}$ 和 $4 \%$ NCC-CPC 的 XRD 图谱中在 $4.8^{\circ}$ 处还出现了 $\mathrm{HA}$ 的 前驱体磷酸八钻 $(\mathrm{OCP})$ 的主极大衍射峰 ${ }^{[20]}$ 。由于 $\mathrm{NCC}$ 的添加量较少, 在 $22.8^{\circ}$ 处没有出现 NCC 主极 大晶面(200)的衍射峰 ${ }^{[21-22]}$ 。

与 $\mathrm{CPC}$ 相比, $2 \% \mathrm{NCC}-\mathrm{CPC}$ 和 4\% NCC-CPC 衍 射峰中 DCPD 和 $\mathrm{CaCO}_{3}$ 主极大晶面的衍射峰强度明 显下降, 而 $\alpha$-TCP 的衍射峰强度下降不明显。分别 以空白 $\mathrm{CPC}$ 中 $\mathrm{DCPD}$ 和 $\mathrm{CaCO}_{3}$ 主极大衍射峰强度 $\left(I_{0}\right)$ 作分母, 以 $2 \%$ NCC-CPC 和 $4 \%$ NCC-CPC 中 $D C P D$ 和 $\mathrm{CaCO}_{3}$ 主极大衍射峰强度 $\left(I_{x}\right)$ 作分子, 进行 归一化处理, 结果如图 3(b)所示。可知加入 NCC 促 进了 DCPD 和 $\mathrm{CaCO}_{3}$ 的溶解和转化, 且 $2 \%$ NCC-CPC 中二相转化量最大。在图 3(a)中, $2 \%$ NCC-CPC 的 XRD 中无明显的 OCP 的衍射峰, 这说 明适量的 NCC 也能够促进中间相 OCP 的溶解和转 化。由于 $\mathrm{DCPD}$ 和 $\mathrm{CaCO}_{3}$ 的溶度积 $K_{\mathrm{sp}}$ 分别为 $1 \times 10^{-7}$ 和 $2.8 \times 10^{-9}$, 较 $\alpha$-TCP 的溶度积 $\left(K_{\mathrm{sp}}=2.0 \times 10^{-29}\right)$ $大^{[21]}$ 。因此, 在 CPC 水化过程中, DCPD 最先溶解, 可释放出 $\mathrm{Ca}^{2+} 、 \mathrm{H}^{+}$和 $\mathrm{PO}_{4}{ }^{3-}, \mathrm{H}^{+}$可促进 $\mathrm{CaCO}_{3}$ 的溶解。 $\mathrm{NCC}$ 表面具有大量亲水性的羟基, 有较强的吸水保 水性, 因此在 $\mathrm{CPC}$ 水化过程中, 随着周围水分的减 

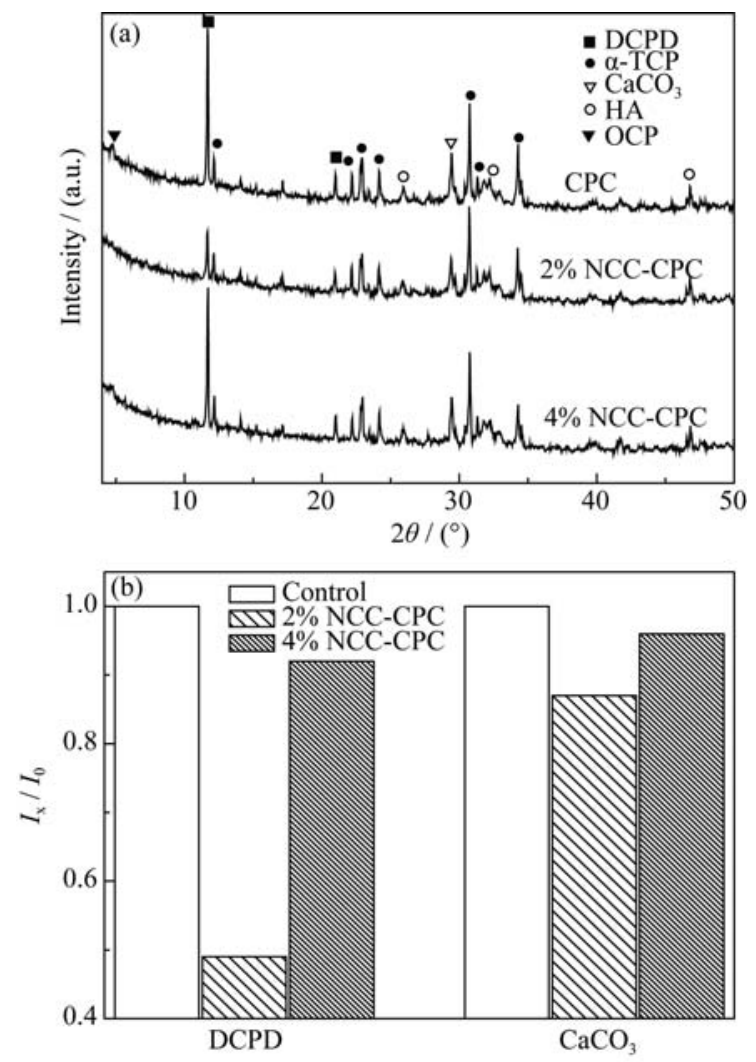

图 $3 \mathrm{CPC} 、 2 \% \mathrm{NCC}-\mathrm{CPC}$ 和 $4 \% \mathrm{NCC}-\mathrm{CPC}$ 水化 $24 \mathrm{~h}$ 后的 $\mathrm{XRD}$ 图谱(a)和各组 CPC 样品中 DCPD 和 $\mathrm{CaCO}_{3}$ 主极大衍射 峰强度 $\left(I_{x}\right)$ 与空白 $\mathrm{CPC}$ 中 $\mathrm{DCPD}$ 和 $\mathrm{CaCO}_{3}$ 主极大衍射峰强度 $\left(I_{0}\right)$ 的比值 $(\mathrm{b})$

Fig. 3 XRD patterns of CPC, 2\% NCC-CPC and 4\% NCC-CPC (a) and the ratio of the primary diffraction maximum of DCPD and $\mathrm{CaCO}_{3}$ of each CPC sample $\left(I_{x}\right)$ to that of $\mathrm{CPC}\left(I_{0}\right)(\mathrm{b})$

少, $\mathrm{NCC}$ 可能释放出吸附水, 加快 DCPD 和 $\mathrm{CaCO}_{3}$ 的溶解。 $\mathrm{NCC}$ 是由 $\mathrm{D}$-吡喃葡萄糖以 $\beta(1-4)$ 糖苷键连 接而成的多糖类物质(化学结构如图 4 所示), 具有 多元醇的性质。Jiang 等 ${ }^{[23]}$ 提出多元醇可与金属阳离 子形成配合物。NCC 分子结构中的每个醇羟基的氧 原子有两对孤电子，而 $\mathrm{Ca}^{2+}$ 可提供空轨道，可与游 离的 $\mathrm{Ca}^{2+}$ 形成配合物, 从而促进式(1)和式(2)所示 的溶解平衡右移。

$$
\mathrm{CaHPO}_{4} \cdot 2 \mathrm{H}_{2} \mathrm{O} \rightleftharpoons \mathrm{Ca}^{2+}+\mathrm{HPO}_{4}{ }^{2-}+2 \mathrm{H}_{2} \mathrm{O}
$$

$$
\mathrm{CaCO}_{3} \rightleftharpoons \mathrm{Ca}^{2+}+\mathrm{CO}_{3}{ }^{2-}
$$

\subsection{CPC 表面元素分析}

图 5 为 $\mathrm{CPC}$ 和 $2 \% \mathrm{NCC}-\mathrm{CPC}$ 水化 $24 \mathrm{~h}$ 后样品 表面的 XPS 图谱。两试样的全谱图均包括 C、O、 $\mathrm{Ca}$ 和 $\mathrm{P}$ 四种元素。与 $\mathrm{CPC}$ 相比, $2 \%$ NCC-CPC 的 $\mathrm{C} 1 \mathrm{~s}$ 峰的强度即 $\mathrm{C}$ 元素含量明显升高。图 5(b d ) 分 别为 $\mathrm{CPC}$ 和 $2 \% \mathrm{NCC}-\mathrm{CPC}$ 的 $\mathrm{C} 1 \mathrm{~s} 、 \mathrm{O} 1 \mathrm{~s}$ 和 $\mathrm{Ca} 2 \mathrm{p}$ 的 高分辨 XPS 图谱，通过高斯拟合，对各峰进行分峰 处理，对应的结合能如表 1 所示。

图 5(b)中 $\mathrm{CPC}$ 在 $288.8 \mathrm{eV}$ 处为 $\mathrm{CaCO}_{3}$ 中 $\mathrm{C}=\mathrm{O}$ 的 $\mathrm{C} 1 \mathrm{~s}$ 结合能 ${ }^{[24]}$, 而 $2 \% \mathrm{NCC}-\mathrm{CPC}$ 该处对应的峰消 失, 在 $288.3 \mathrm{eV}$ 出现新峰, 属于 $\mathrm{NCC}$ 结构中糖苷键 $\mathrm{C}-\mathrm{O}-\mathrm{C}$ 的结合能 ${ }^{[25-26]}$ 。图 5(c)中 $\mathrm{CPC}$ 在 $532.5 \mathrm{eV}$ 处出现 DCPD 中 $\mathrm{H}_{2} \mathrm{O}$ 的 $\mathrm{O} 1 \mathrm{~s}$ 结合能，而 $2 \% \mathrm{NCC}$ $\mathrm{CPC}$ 此峰消失, 在 $532.8 \mathrm{eV}$ 处出现新峰, 属于 $\mathrm{NCC}$ 中 $\mathrm{C}-\mathrm{OH}$ 和 $\mathrm{C}-\mathrm{O}-\mathrm{C}$ 的 $\mathrm{O} 1 \mathrm{~s}$ 结合能 ${ }^{[27-28]}$ 。图 5(d)中 347.3 和 $351.0 \mathrm{eV}$ 处分别属于 $\mathrm{Ca} 2 \mathrm{p} 1 / 2$ 和 $\mathrm{Ca} 2 \mathrm{p} 3 / 2$ 的结合能 ${ }^{[26]}$,与 CPC 相比, $2 \%$ NCC-CPC 的 Ca2p $1 / 2$ 结合能发生 $0.3 \mathrm{eV}$ 的化学位移。如 $\mathrm{Ca}^{2+}$ 与 $\mathrm{NCC}$ 之 间发生配位结合, $\mathrm{Ca} 2 \mathrm{p}$ 的结合能应减小, 但实际却 增大 $0.3 \mathrm{eV}$, 可能是由于 $\mathrm{NCC}$ 分子结构中众多羟基 氧和吡喃环上的氧强的电负性使得与 $\mathrm{Ca}^{2+}$ 配位的羟 基上的电子云密度偏离钫，导致 $\mathrm{Ca} 2 \mathrm{p}$ 的结合能增 大，这也暗示了 $\mathrm{NCC}$ 与 $\mathrm{Ca}^{2+}$ 形成的配合物不稳定， 会向稳定相转化。

\subsection{CPC 断面微观形貌分析}

图 6 为各组 CPC 水化 $24 \mathrm{~h}$ 后自然断面的 SEM 照片。可见, CPC 断面存在较多的孔隙和裂纹, 且颗 粒尺寸较大，颗粒间排列较疏松。而加入 $2 \%$ 和 $4 \%$ NCC 的 CPC 断面孔隙和裂纹相对较少, 颗粒细化, 颗粒间排列较致密。

抗压强度是衡量 CPC 固化后抗负载能力的指 标，是材料产生裂纹并延伸至临界裂纹尺寸时所承 受的最大负荷。作为脆性材料, CPC 在受荷时首先会 在颗粒结合处产生微裂纹, 当微裂纹与 CPC 内部孔 隙相遇时会并接而使裂纹迅速生长, 从而影响 $\mathrm{CPC}$

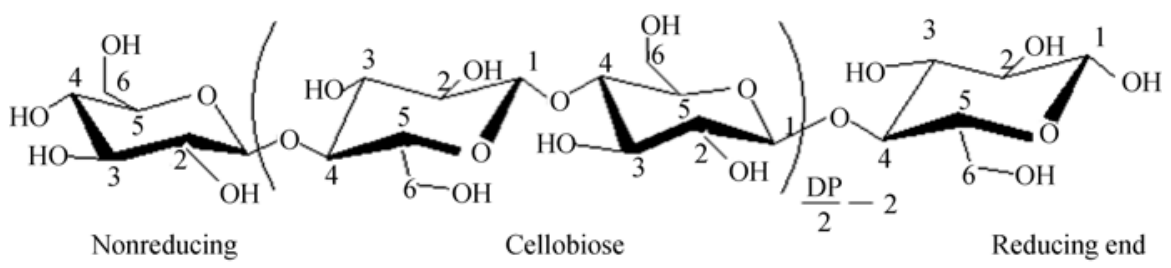

图 4 NCC 的化学结构图(其中 DP 为聚合度 ${ }^{[8]}$ )

Fig. 4 Chemical structure of NCC DP represents the degree of polymerization 

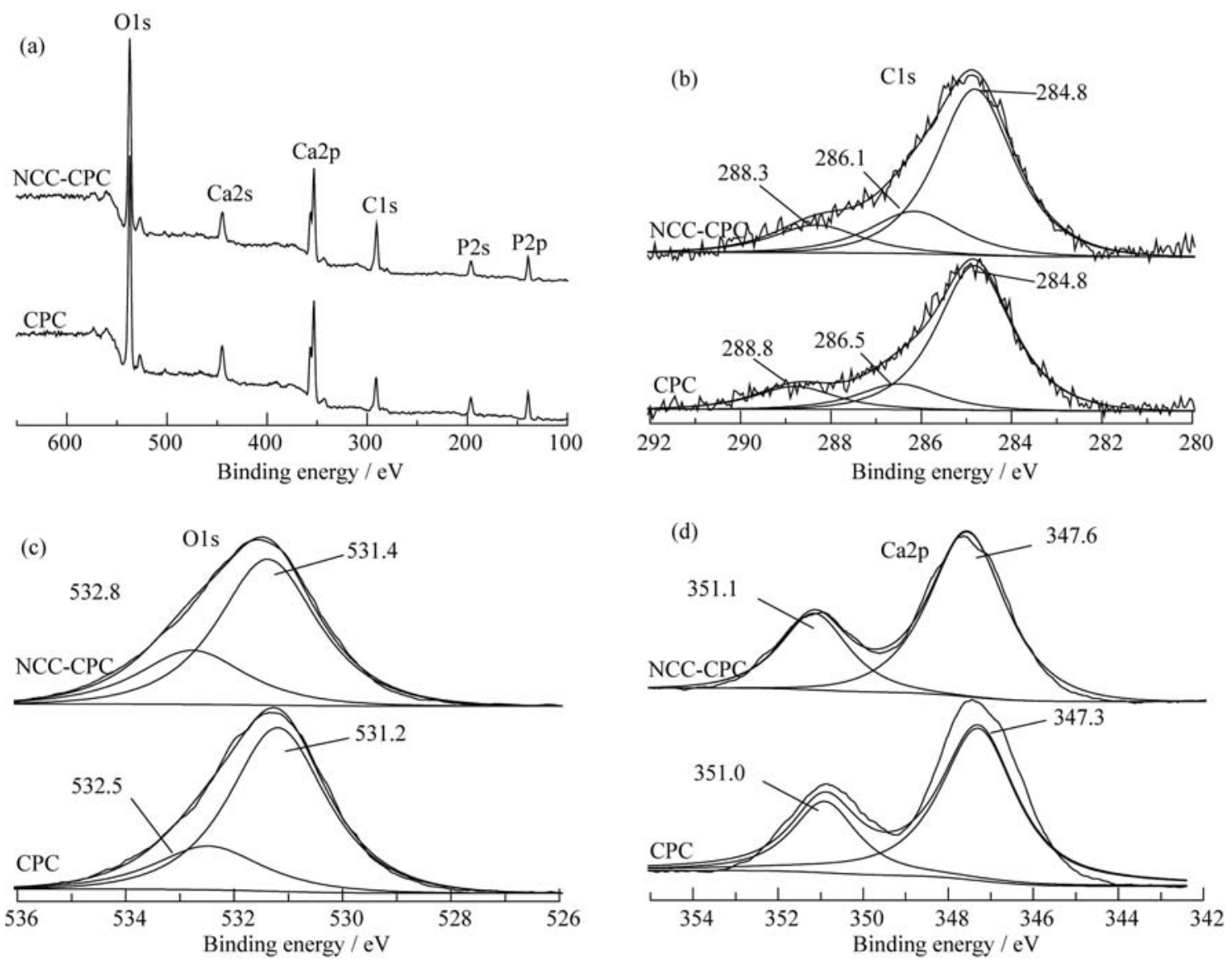

图 $5 \mathrm{CPC}$ 和 $2 \% \mathrm{NCC}-\mathrm{CPC}$ 水化 $24 \mathrm{~h}$ 后的 XPS 图谱

Fig. 5 XPS spectra of CPC and 2\% NCC-CPC after setting for $24 \mathrm{~h}$ (a) Survey; (b) $\mathrm{C} 1 \mathrm{~s}$; (c) O1s; (d) Ca2p

的抗压强度。因此, CPC 的抗压强度取决于其颗粒 间的结合强度、孔隙和裂纹的特征等。

综上, $\mathrm{CPC}$ 在水化过程中, $\mathrm{DCPD}$ 和 $\mathrm{CaCO}_{3}$ 由于 溶度积较大首先溶解产生 $\mathrm{Ca}^{2+}$; $\mathrm{NCC}$ 表面羟基与游 离的 $\mathrm{Ca}^{2+}$ 形成正离子配合物, 促进 DCPD 和 $\mathrm{CaCO}_{3}$ 的溶解，但该配合物稳定性较差; 配合物吸附带负 电的 $\mathrm{PO}_{4}{ }^{3-}, \mathrm{PO}_{4}{ }^{3-}$ 再吸附 $\mathrm{Ca}^{2+}$, 如此循环形成弱结晶 的中间相，使得 $\mathrm{CPC}$ 颗粒之间排列紧密，孔隙减少，

\section{表 1 CPC 和 2\% NCC-CPC 中 C1s、O1s 和 Ca2p 的结合能 \\ Table 1 The binding energy of $\mathrm{C} 1 \mathrm{~s}, \mathrm{O} 1 \mathrm{~s}$ and $\mathrm{Ca} 2 \mathrm{p}$ core levels from CPC and 2\% NCC-CPC}

\begin{tabular}{cccc}
\hline \multirow{2}{*}{$\begin{array}{c}\text { Elements and functional } \\
\text { groups }\end{array}$} & \multicolumn{2}{c}{ Binding energy/eV } \\
\cline { 2 - 4 } & $\mathrm{C}-\mathrm{C} 、 \mathrm{C}-\mathrm{H}$ & 284.8 & $2 \%$ NCC-CPC \\
\hline \multirow{2}{*}{$\mathrm{C} 1 \mathrm{~s}$} & $\mathrm{C}-\mathrm{O} / \mathrm{C}=\mathrm{O}$ & $286.5 / 288.8$ & 284.8 \\
& $\mathrm{C}-\mathrm{C} / \mathrm{C}-\mathrm{O}-\mathrm{C}$ & - & - \\
\hline \multirow{2}{*}{$\mathrm{O} 1 \mathrm{~s}$} & $\mathrm{P}=\mathrm{O}$ & 531.2 & 531.4 \\
& $\mathrm{P}-\mathrm{OH} / \mathrm{H}_{2} \mathrm{O}$ & 532.5 & - \\
& $\mathrm{C}-\mathrm{OH} / \mathrm{C}-\mathrm{O}-\mathrm{C}$ & - & 532.8 \\
\hline \multirow{2}{*}{$\mathrm{Ca} 2 \mathrm{p}$} & $\mathrm{Ca} 2 \mathrm{p}_{3 / 2}$ & 351.0 & 351.1 \\
& $\mathrm{Ca} 2 \mathrm{p}_{1 / 2}$ & 347.3 & 347.6 \\
\hline
\end{tabular}

" - " indicates the absence of binding energy corresponding to the element
图 7 为该过程的示意图。同时 $\mathrm{NCC}$ 延长了 $\mathrm{CPC}$ 的 凝固时间，即保持良好流动性的时间延长，从而有 利于内部气体的排出, 使得结构更加紧密 ${ }^{[21]}$ 。本研 究添加的 NCC 长为 150 200 nm, 宽为 10 15 nm(图 8 为 NCC 的 TEM 照片), 具有较高的长径比, 类似 于晶须, 当 $\mathrm{CPC}$ 在抵抗应力破坏时, 具有高强度的 NCC 可能通过桥接作用, 在裂纹表面上施加闭合应 力, 从而减小裂纹尖端所承受的力, 起到抑制裂纹 继续扩展而增加 $\mathrm{CPC}$ 的断裂韧性 ${ }^{[29]}$ 。上述原因可能 提高 $\mathrm{CPC}$ 的抗压强度。

\subsection{NCC 在 CPC 中的分散性研究}

图 9 为 $\mathrm{AMC}$ 标记的 $\mathrm{NCC}$ 在 $\mathrm{CPC}$ 中分布的荧 光显微照片。AMC 标记的 $\mathrm{NCC}$ 呈绿色荧光, 随 NCC 含量的增加, 荧光强度增强, 而未经 AMC 标 记的 NCC 加入 CPC 后, 无绿色荧光。带有绿色荧 光的颗粒在 CPC 中的分布均匀, 说明 NCC 在 CPC 中分散较均匀。

\section{3 结论}

1) 加入 NCC 显著提高 $\mathrm{CPC}$ 的抗压强度和断裂 

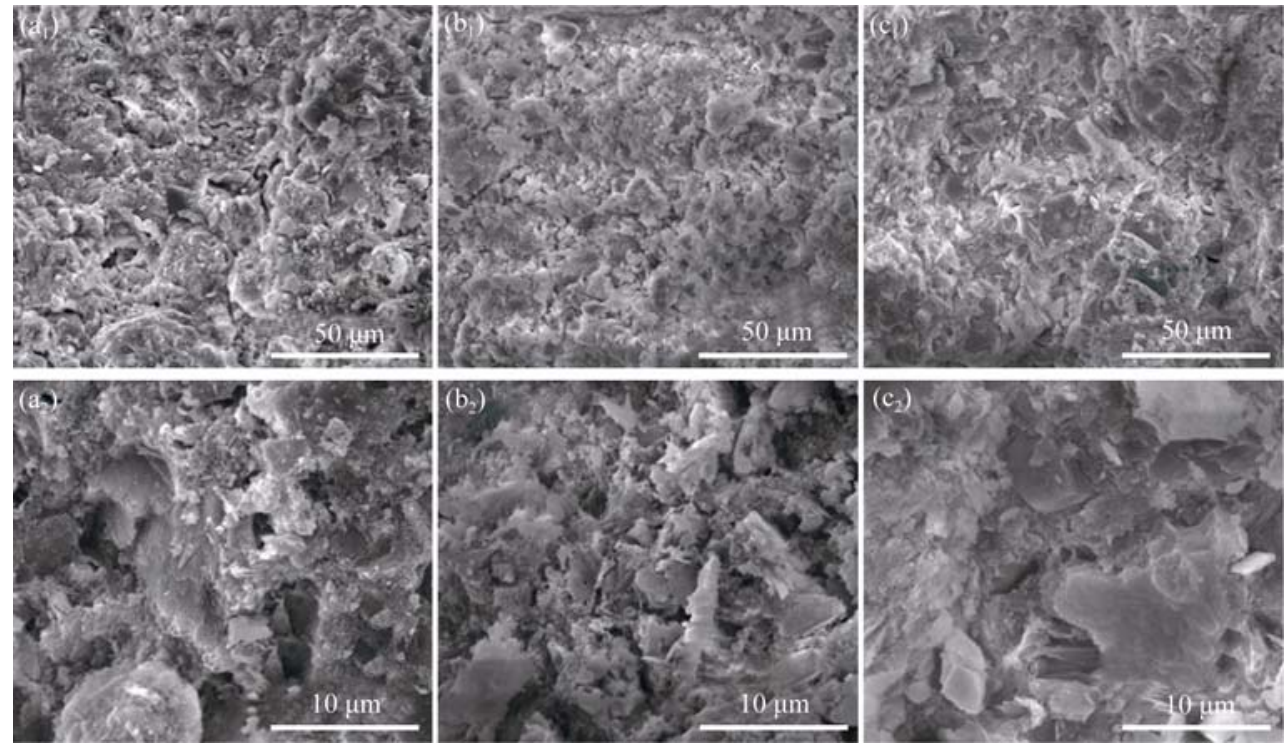

图 6 CPC、2\% NCC-CPC 和 4\% NCC-CPC 水化 $24 \mathrm{~h}$ 后自然断面的 SEM 照片

Fig. 6 SEM images of the cross-sections of CPC $\left(a_{1}, a_{2}\right), 2 \%$ NCC-CPC $\left(b_{1}, b_{2}\right)$ and 4\% NCC-CPC $\left(c_{1}, c_{2}\right)$ after setting for $24 \mathrm{~h}$

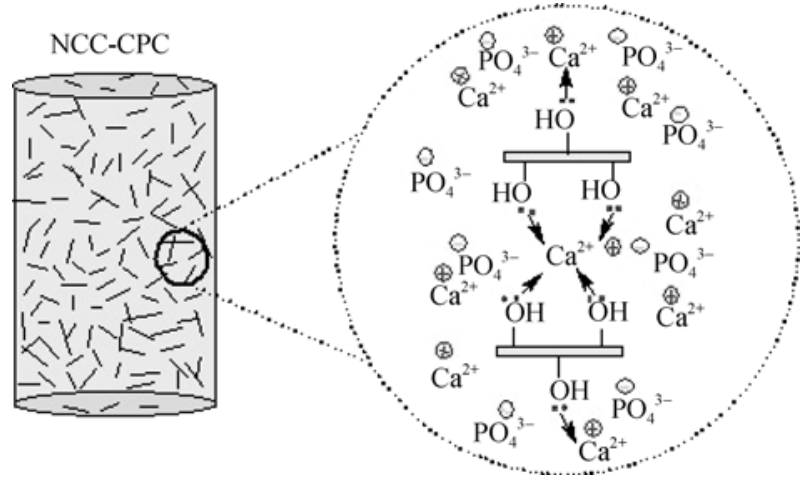

图 $7 \mathrm{NCC}$ 与 $\mathrm{Ca}^{2+}$ 配位结合促进 $\mathrm{CPC}$ 水化的示意图

Fig. 7 Schematic diagram of NCC promoting the setting of $\mathrm{CPC}$ by coordination with $\mathrm{Ca}^{2+}$
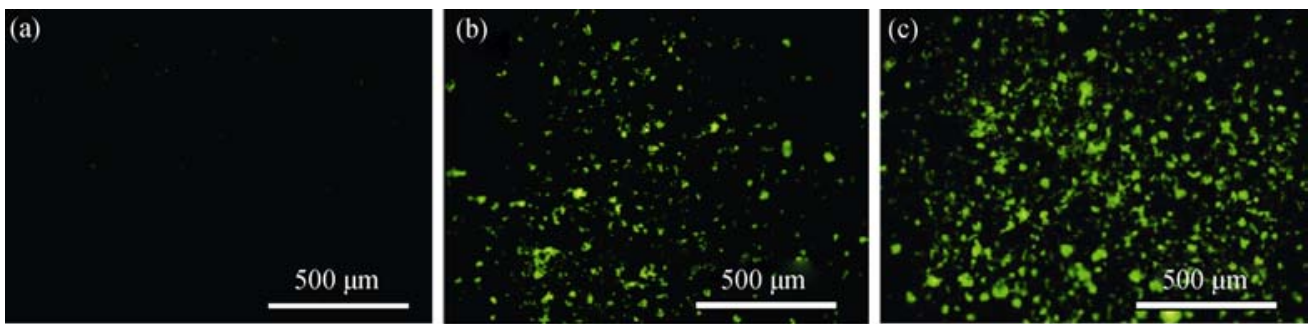

图 9 7-氨基-4-甲基香豆素(AMC)标记的 $\mathrm{NCC}$ 在 $\mathrm{CPC}$ 中均匀分布的荧光显微照片

Fig. 9 Fluorescence micrographs of NCC labeled by 7-amino-4-methyl coumarin (AMC) that evenly distributed in CPC (a) $2 \%$ NCC-CPC; (b) $2 \%$ NCC-AMC-CPC; (c) $4 \%$ NCC-AMC-CPC

㓞性，且 $2 \%$ NCC-CPC 的抗压强度最高，为 $(26.9 \pm$ 3.4) $\mathrm{MPa}$ 。同时 NCC 延长 CPC 的凝固时间, $2 \%$ $\mathrm{NCC}-\mathrm{CPC}$ 的凝固时间基本满足临床需求;

2) XRD 和 XPS 结果显示, $\mathrm{NCC}$ 与 $\mathrm{Ca}^{2+}$ 形成不 稳定的配合物, 促进了 $\mathrm{DCPD}$ 和 $\mathrm{CaCO}_{3}$ 的溶解和转 化。SEM 照片显示加入 NCC 后, CPC 中孔隙和裂纹
减少，颗粒尺寸减小。 $\mathrm{NCC}$ 具有高长径比，可能对 裂纹具有桥接作用，起到补强增韧的作用;

3) 荧光素 AMC 标记的 NCC, 表明在 CPC 中呈 均匀分布。

本研究通过添加 $\mathrm{NCC}$, 提高了 $\mathrm{CPC}$ 的抗压强度 和韧性, 为 $\mathrm{CPC}$ 的补强增㓞提供了新方法, 具有潜 
在的应用前景。

\section{参考文献:}

[1] TAKAGI S, CHOW L C, ISHIKAWA K. Formation of hydroxyapatite in new calcium phosphate cements. Biomaterials, 1998, 19: $1593-1599$

[2] SAWAMURA T, MIZUTANI Y, OKUYAMA M, et al. Setting time and formability of calcium phosphate cements prepared using modified dicalcium phosphate anhydrous powders. J. Mater. Sci. Mater. Med., 2014, 25(7): 1631-1636.

[3] HEINEMANN S, ROSSLER S, LEMM M, et al. Properties of injectable ready-to-use calcium phosphate cement based on waterimmiscible liquid. Acta Biomater., 2013, 9(4): 6199-6207.

[4] LIU W, ZHANG J, RETHORE G, et al. A novel injectable, cohesive and toughened Si-HPMC (silanized-hydroxypropyl methylcellulose) composite calcium phosphate cement for bone substitution. Acta Biomater., 2014, 10(7): 3335-3345.

[5] MOHAMMADI M, HESARAKI S, HAFEZI-ARDAKANI M. Investigation of biocompatible nanosized materials for development of strong calcium phosphate bone cement: comparison of nano-titania, nano-silicon carbide and amorphous nano-silica. $\mathrm{Ce}$ ram. Int., 2014, 40(6): 8377-8387.

[6] PENG B L, DHAR N, LIU H L, et al. Chemistry and applications of nanocrystalline cellulose and its derivatives: a nanotechnology perspective. Can. J. Chem. Eng., 2011, 89(5): 1191-1206.

[7] MOON R J, MARTINI A, NAIRN J, et al. Cellulose nanomaterials review: structure, properties and nanocomposites. Chem. Soc. Rev., 2011, 40(7): 3941-3994.

[8] HABIBI Y, LUCIA L A, ROJAS O J. Cellulose nanocrystals: chemistry, self-assembly, and applications. Chem. Rev., 2010, 110(6): $3479-3500$.

[9] WU W B, ZHANG L. Functionalization and application of nanocrystalline cellulose. Progress Chem., 2014, 26: 403-414.

[10] KLEMM D, KRAMER F, MORITZ S, et al. Nanocelluloses: a new family of nature-based materials. Angew. Chem. Int. Ed., 2011, 50: $5438-5466$.

[11] ZHANG R, ZHU C, SHAN X, et al. Study on the poly (3-hydroxybutyrate-co-4-hydroxybutyrate)-based nanocomposites reinforced by surface modified nanocrystalline cellulose. J. Appl. Polym. Sci., 2013, 130(3): 2015-2022.

[12] JIANG X X, QU S X, LIN S Z, et al. Influnence of drynaria on physicochemical and in vitro biological properties of calcium phosphate cement. J. Inorg. Mater., 2010, 26(1): 29-37.

[13] OREFFO R O C, DRIESSENS F, PLANELL J A, et al. Growth and differentiation of human bone marrow osteoprogenitors on novel calcium phosphate cements. Biomaterials, 1998, 19(20): $1845-1854$.
[14] HUANG J L, LI C J, GRAY D G. Cellulose nanocrystals incorporating fluorescent methylcoumarin groups. ACS Sustainable Chem. Eng., 2013, 1(9): 1160-1164.

[15] KHAIROUN I, DRIESSENS F C M, BOLTONG M G, et al. Addition of cohesion promotors to calcium phosphate cements. Biomaterials, 1999, 20(4): 393-398.

[16] LALIA B S, GUILLEN E, ARAFAT H A, et al. Nanocrystalline cellulose reinforced PVDF-HFP membranes for membrane distillation application. Desalination, 2014, 332(1): 134-141.

[17] HOENICH N A. Cellulose for medical applications: past, present, and future. Biorsources, 2007, 1(2): 270-280.

[18] KHAIROUN I, BOLTONG M G, DRIESSENS F C M, et al. Effect of calcium carbonate on clinical compliance of apatitic calcium phosphate bone cement. J. Biom. Mater. Res., 1997, 38(4): 356-360.

[19] SHEN W, LIU C S, GU Y F. Hydration reaction, setting time and compressive strength of calcium phosphate cement. J. Chin. Ceram. Soc., 1998, 26(2): 129-135.

[20] SUZUKI O. Octacalcium phosphate: osteoconductivity and crystal chemistry. Acta Biomater., 2010, 6(9): 3379-3387.

[21] TANG Y, YANG S, ZHANG N, et al. Preparation and characterization of nanocrystalline cellulose via low-intensity ultrasonic-assisted sulfuric acid hydrolysis. Cellulose, 2013, 21(1): 335-346.

[22] LI M H, QU S X, YAO N, et al. Properties of calcium phosphate cement with various concentrations of Xiangdan injection. $J$. Inorg. Mater., 2010, 25(5): 507-511.

[23] JIANG C Y, ZHANG N, GAO X W. Assistanted stabilizer in environment-friendly $\mathrm{Ca} / \mathrm{Zn}$ composite stabilizer. Mod. Plast. Process Appl., 2008, 20(2): 61-63.

[24] NI M, RATNER B D. Differentiating calcium carbonate polymorphs by surface analysis techniques-an XPS and TOF-SIMS study. Surf. Interface Anal., 2008, 40(10): 1356-1361.

[25] MATUANA L M, BALATINECZ J J, SODHI R N S, et al. Surface characterization of esterified cellulosic fibers by XPS and FTIR Spectroscopy. Wood Sci. Technol., 2001, 35(3): 191-201.

[26] JOHANSSON L S, CAMPBELL J M. Reproducible XPS on biopolymers: cellulose studies. Surf Interface Anal, 2004, 36(8): $1018-1022$

[27] CHUSUEI C C, GOODMAN D W, VAN STIPDONK M J, et al. Calcium phosphate phase identification using XPS and time-of-flight cluster SIMS. Anal. Chem., 1999, 71(1): 149-153.

[28] MAACHOU H, GENET M J, ALIOUCHE D, et al. XPS analysis of chitosan- hydroxyapatite biomaterials: from elements to compounds. Surf. Interface Anal., 2013, 45(7): 1088-1097.

[29] LING G Y, WU B Y, LEI T Q, et al. A review on the reinforcing and toughening mechanisms of whisker reinforcing ceramic matrix composites. J. South China Univ. Technol., 1996, 24(7): 34-39. 